\title{
ABSTRACTS OF THE 2019 SUMMIT ON NATIONAL AND GLOBAL CANCER HEALTH DISPARITIES (SCHD19)
}

\author{
27-28 April, 2019, \\ Issaquah, WA, USA
}

\section{Organized by}

The Binaytara Foundation 
1

\section{COST-EFFECTIVE CARE FOR PATIENTS NEWLY DIAGNOSED WITH BREAST CANCER: THINK GLOBALLY, ACT LOCALLY}

Kevin B. Knopf, Shannon Ugarte, Annie Tang, Zhonnet Harper and Amal Khoury

Highland Hospital, Oakland, CA, U.S.A.

Background: Conscientious use of scarce health economic resources allows for social distributive justice by avoiding overtreatment of patients in order to allow for appropriate treatment for a broader patient population. Patients with breast cancer with lower socioeconomic status have a worse outcome compared to those with higher socioeconomic status in the United States. In part, this is due to poor access to medical care and a failure of logistical care. Materials and Methods: We implemented a community-based breast cancer multidisciplinary program with weekly meetings to discuss patients, coordinate care, and provide cost-effective care to a medically underserved and vulnerable patient population. Breast surgery and medical oncology saw patients together in a co-located space and expedited timely initiation of neoadjuvant and adjuvant chemotherapy/hormone therapy for appropriate patients. The conjoint clinic allowed for rapid temporal integration of care in our safety net hospital/community. A single breast cancer navigator provided rapid identification of patients, coordination through clinics, and assistance with social work and other needs. Weekly 30-minute discussion sessions reviewing all new and relevant follow-up patient issues were implemented in lieu of a Breast Cancer Tumor Board for efficiency. The relevant published clinical literature was reviewed on an ongoing basis. Results/Conclusion: Cost effective care was obtained by implementing: i) Avoidance of post lumpectomy radiation for selected patients based on PRIME2 trial results; ii) judicious use of postmastectomy radiation based on a careful re-analysis of the data; iii) avoidance of adjuvant/neoadjuvant Perjeta in patients with human epidermal receptor growth factor receptor 2 positive disease based on a lack of overall survival; iv) adoption of 6-month schedule of adjuvant Herceptin; v) avoidance of excessive imaging by following National Comprehensive Cancer Network guidelines and clinical judgment; and vi) limiting dose-dense chemotherapy to appropriate patients (those with estrogen receptor-negative high-risk node-negative or nodepositive disease).

\section{2}

A QUESTIONNAIRE-BASED COMMUNITY OUTREACH STUDY TO DEFINE THE VARIOUS FACTORS BEHIND HIGHER INCIDENCE AND

\section{POORER OUTCOMES OF BREAST CANCER IN AFRICAN-AMERICAN WOMEN IN BROOKLYN}

\author{
Alankrita Taneja, Lynette Leepack and \\ Donna Marie Mannaseh \\ Maimonides Medical Centre, Brooklyn, NY, U.S.A.
}

Background: Various studies have correlated the variation in the incidence and outcomes of breast cancer to racial and ethnic differences. Particularly in the United States, several studies have found that breast cancer in African-American women tends to be more aggressive compared to their Caucasian counterparts. While a genetic component might predispose this group to poorer survival rates, there is also an undefined cultural, socioeconomic and lifestyle component to it. Several studies have tried to study the various factors influencing the incidence of outcomes of breast cancer in African-American women, however, these factors are still poorly defined. With our study, we aim to use a questionnaire-based community outreach study in the Flatbush area in Brooklyn to define these factors. Furthermore, our goal is to target the major factors we elucidate in order to increase the knowledge, attitude and practices of these women towards breast cancer awareness. Materials and Methods: The Flatbush area in Brooklyn has been identified as being densely populated with AfricanAmerican communities using demographic surveys. Partnerships will be formed with community-based organizations with predominance of African-American women such as church groups. A community-based needs assessment will be performed using a validated questionnaire to assess the demographics, screening history, and barriers to screening and seeking treatment in African-American women. A team comprising resident physicians and nurse practitioners will be involved in gathering the data. Descriptive statistics will be used to summarize and analyze data. Expectations: Over a 12-month period between January to December 2019, 100 African-American women will be surveyed to assess the role of various factors including cultural, socioeconomic and lifestyle factors in poorer outcomes in breast cancer incidence and survival. While it is speculated that there is an interplay of various factors in poorer breast cancer outcomes in this population, there is still no clear objective data about the weightage of each of these factors as compared to genetic components. Conclusion: Given the diverse population in Brooklyn, we believe that our study will be able to assess the situation better and help us devise a tailored breast health education and outreach program for this population in the Flatbush area of Brooklyn. Understanding this question and the clear significance of genetic component will also play a role in the Precision Medicine initiative by National Institutes of Health. 
3

\section{DISPARITIES IN TREATMENT OF BRAIN METASTATIC BREAST CANCER WITH STEREOTACTIC RADIOSURGERY AND WHOLE-BRAIN RADIATION: INSIGHTS FROM THE NCDB DATABASE}

Muhammad Salman Faisal ${ }^{1}$, Ahmed Khattab ${ }^{1}$, Stephen $\mathrm{Abel}^{2}$, Shaakir Hasan ${ }^{2}$, Abhishek Chaturvedi ${ }^{1}$ and Rodney Wegner ${ }^{2}$

${ }^{1}$ Department of Internal Medicine, Allegheny

Health Network, Pittsburgh, PA, U.S.A.;

${ }^{2}$ Department of Radiation Oncology, Allegheny

Health Network, Pittsburgh, PA, U.S.A.

Background: Breast cancer is the most common malignancy in women, accounting for over 300,000 cases per year. Unfortunately, brain metastases are found in a sub-group of patients with breast cancer, even at presentation. Radiation treatment is used for brain metastasis and can be delivered by whole-brain radiation therapy (WBRT) or by more focused stereotactic radiation surgery (SRS). We utilized the National Cancer Database (NCDB) to analyze the incidence of brain metastases at diagnosis in breast cancer patients, as well as trends in radiation use/technique. Materials and Methods: The NCDB was queried for patients who were diagnosed with breast cancer between 2004-2015 and had brain metastasis at presentation $(\mathrm{N}=4,491)$. Patients without brain radiation and with inadequate follow-up were excluded. Odds ratios were calculated to identify factors associated with treatment. Multivariable Cox regression was used to determine predictors of survival. The final cohort consisted of 1,505 patients. Results: Using the eligibility criteria above 1,505 patients were identified in the NCDB. The cohort had a mean age of 58 years and, as expected, was overwhelmingly female (99.3\%). A small proportion of this population was uninsured (7\%) and $81 \%$ of radiation treatment was received at cancer centers located in metropolitan areas. Two hundred and sixty-one (17.3\%) patients underwent SRS, while 1,244 (82.7\%) received WBRT. Those patients with private insurance, higher income, metropolitan location, and having care delivered at an academic center were more likely to receive SRS. Conversely, the likelihood of receiving WBRT was significantly higher in those with luminal type cancer, African-Americans, the uninsured, and those located in urban areas or treated at a community cancer center. Conclusion: This analysis of the NCDB highlights disparities across socioeconomic factors as it relates to the delivery of more advanced radiation techniques. There is a continued need to reduce these disparities and improve access to care for at-risk populations affected by this common malignancy.
4

\section{UPDATE ON SPOT ON CML}

Olga Sala Torra, Lan Beppu, Catherine Felton, Luke Monroe and Jerald P. Radich

Fred Hutchinson Cancer Research Center, Seattle, WA, U.S.A.

Background: All chronic myeloid leukemias (CML) have the chimeric breakpoint cluster region-abelson (BCR-ABL) fusion gene resulting from the $t(9 ; 22)$ chromosomal abnormality. $B C R-A B L$ mRNA is the basis of reverse transcriptionpolymerase chain reaction (RT-PCR) assays for diagnosis and monitoring. Tyrosine kinase inhibitors (TKIs) can target BCR$\mathrm{ABL}$ and drastically improve outcome for patients with CML. Optimal care in CML requires peripheral blood monitoring of $B C R-A B L$ in order to assess response, and mutation analysis of ABL's tyrosine kinase domain if resistance to TKIs develops. Access to TKIs and molecular diagnostics is limited in developing countries. 'Spot on CML', a collaboration between the Fred Hutchinson Cancer Research Center, The Max Foundation, Cepheid, and the International CML Foundation, started in September 2017 to facilitate access to testing for CML to developing countries. In some countries, patients diagnosed with CML can receive free TKI treatment through Max Foundation's partnership with Novartis and other pharmaceutical companies. Materials and Methods: Patients' dried blood spots (DBS) are sent to the Fred Hutchinson Cancer Research Center. Testing for $B C R-A B L$ mRNA is carried out using lysate and Cepheid GeneXpert platform. $A B L$ mutations are assayed by Sanger sequencing. We demonstrated the accuracy of DBS in head-to-head comparisons with standard peripheral blood testing (1). Results: Since September 2017, we have received specimens for 463 patients from 21 institutions in 19 countries in Central and South America, Asia, Africa and Oceania. A total of 449 specimens were submitted for detection/quantification of $B C R-A B L$ and 12 for mutation analysis, with all specimens proving adequate for analysis. Overall, 348 specimens were positive for $B C R-A B L$ (75.3\%) at levels ranging from $0.0038 \%$ to $110 \%$. No $A B L$ mutations were detected. Regular updates for this project are posted on: https://spotoncml.shinyapps.io/SPOTonCML/. Conclusion: SPOT on CML is a collaboration that effectively provides testing for CML in developing countries. The use of DBS is cost-effective: batches of samples can be sent to a central laboratory by slow but inexpensive transportation.

1 Sala Torra O, Beppu L, Smith JL, Welden L, Georgievski J, Gupta K, Kumar R, Yeung CC, Paguirigan A, Gooley TA, Branford S and Radich JP: Paper or plastic? BCR$A B L 1$ quantitation and mutation detection from dried blood spots. Blood 127: 2773-2774, 2016. PMID: 27076173. DOI: 10.1182/blood-2015-12-689059 
5

\section{5-YEAR OVERALL SURVIVAL OUTCOMES FOR PATIENTS WITH BREAST CANCER TREATED AT THE WEST CANCER CENTER BETWEEN 2007-2009}

Gregory Vidal, Axel Grothey, Brad Somer and Lee Schwartzberg

West Cancer Center, Memphis, TN, U.S.A.

Background: Despite advances in breast cancer treatment, disparities in outcome exist. Memphis is reported to have one of the largest disparities in breast cancer mortality between African-American and Caucasian patients. To fully address this difference, oncology practices will need to evaluate their practice patterns and outcomes. Here we report 5 -year overall survival (OS) data for the West Cancer Center and Research Institute (WCCRI) for the years 2008 and 2009. Materials and Methods: A retrospective analysis was performed utilizing ICD-9 codes to identify potential subjects, then electronic medical record data, physician progress notes, path reports and social security data were cross-referenced. Dates of death data were verified in the social security death index. To qualify, subjects were required to have at least 30 days of follow-up and at least one evaluation and management visit. Results: In 2008 and 2009, a total of 595 and 726 subjects were analyzed respectively (Table I). For 2008 and 2009 combined, there were more Caucasian than African-American patients with stage I $(p=0.076)$ and with stage IV $(p=0.06)$ disease. The 5 -year OS rate was around $10 \%$ lower for African-American compared with Caucasian patients. Stage-by-stage, there were no major differences in the 5-year OS rate, with exceptions of stage II in 2009 and stage III in 2008 (both favoring Caucasians). Median annual income was significantly higher in Caucasians than Backs, and this difference widened from 2008 to 2009 . The 5-year OS rate was dependent on annual income, with patients in the lowincome bracket having significantly poorer outcome. Those with triple-negative breast cancer, grade 3 disease, age $>70$ years and median income less than $\$ 40,000$ had a significantly lower survival rate. Outcomes for patients treated at the WCCRI met or surpassed Surveillance Epidemiology and End Results reported data. Conclusion: Disparities in 5-year OS do exist along racial, biological and socioeconomic lines. A multi-pronged approach will be required to achieve equipoise in outcome.

Table I. West Cancer Center and Research Institute breast cancer outcomes.

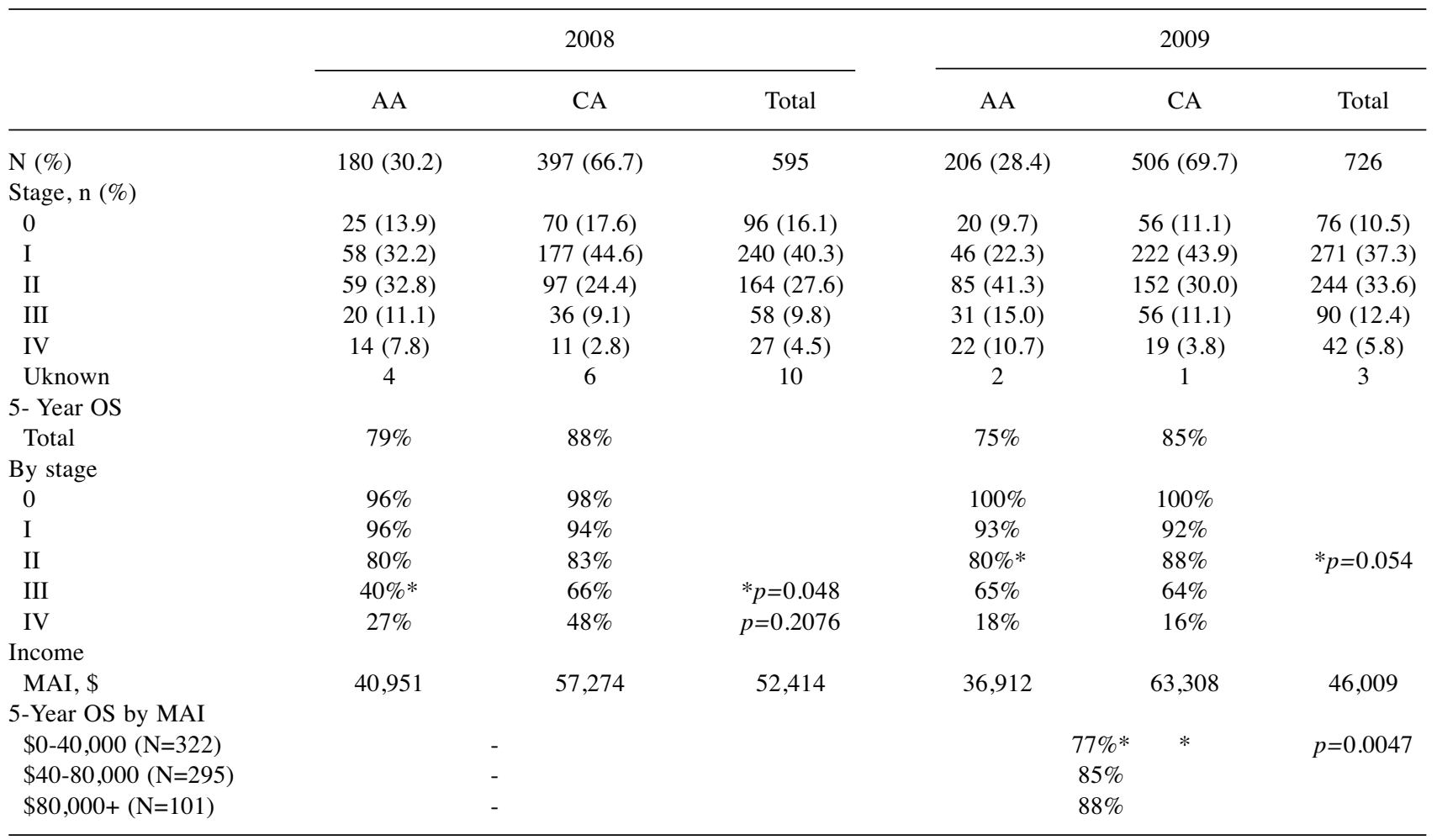

AA: African-American; CA: Caucasian; MAI: median annual income; OS: overall survival. Median follow-up of 94 months. 
6

\section{OVERDIAGNOSIS OF THYROID CANCER: A PROBLEM OF GREATER ACCESS TO HEALTHCARE}

\author{
Janeesh Sekkath Veedu ${ }^{1}$ and Emily Sekkath Veedu ${ }^{2}$ \\ ${ }^{1}$ Department of Internal Medicine, University \\ of Kentucky, Lexington, KY, U.S.A.; \\ ${ }^{2}$ Markey Cancer Center, University of \\ Kentucky, Lexington, KY, U.S.A.
}

Background: Access to cancer care is a major problem worldwide, leading to poor outcomes. However, overdiagnosis due to improved access is an ignored phenomenon. The incidence of differentiated thyroid cancer, especially papillary, has been rising worldwide, including India. A large proportion of this is attributed to the phenomenon of overdiagnosis. Materials and Methods: We analyzed the incident rate of thyroid cancers using data from Population Based Cancer Registries compiled by the National Cancer Registry Program of the Government of India from 14 regions which reported data from 2004/05 to 2013/14. Results: There was a relative increase of $62 \%$ and $48 \%$ in incidence rate of thyroid cancer in women and men, respectively. The relative increase was highest in age groups $<30$ years $(121 \%)$ and $30-44$ years $(107 \%)$, and similarly in men of age group $<45$ years. In Kerala state in India, there was a $93 \%$ increase in incidence over a decade. The incidence was four-fold higher there compared with other regions, but the mortality rate remained stable, suggesting overdiagnosis as the most plausible etiology. Conclusion: The dramatic rise in incidence of thyroid cancer in India is likely due to overdiagnosis. Overdiagnosis was a problem in developed nations but with changing epidemiology and greater access to care, this phenomenon is also occurring in developing countries. India is seeing a mushrooming of diagnostic laboratories and imaging modalities. Kerala with its high socioeconomic and educational status has healthcare access and quality of a developed region. This is probably the driver for the high incidence of thyroid cancer in the state. Access to healthcare can be a double-edged sword.

\section{7}

\section{ESTABLISHMENT OF THE BINAYTARA FOUNDATION CANCER CENTER IN PROVINCE 2 OF NEPAL}

\section{Binay Shah, Tara Shah and Derrel Walker}

Binaytara Foundation, Issaquah, WA, U.S.A.

Background: Like the rest of South Asia, the low-income country of Nepal is experiencing a surging burden of noncommunicable diseases such as cancer. All of the comprehensive cancer centers are currently located in one province, Province 3. We describe our experience establishing a 25-bed cancer hospital in Province 2 (population 5.5 million) of Nepal. Materials and Methods: This project is a collaborative effort of the Binaytara Foundation (applicant organization), and the Binaytara Cancer Trust (the Binaytara Foundation's sister organization in Nepal). We rented a building for the hospital in Janakpur, Nepal. Following the Government of Nepal's guidelines, we received government approval to operate a 25-bed hospital. The hospital has an emergency room, 24-hour pharmacy, laboratory, outpatient clinics and an inpatient ward. A physician was trained to provide palliative care. Training included webinars as well as 2 weeks of onsite training by a palliative care physician. A surgeon and an oncologist were hired to provide surgery and chemotherapy services. Results: The hospital currently provides palliative care, surgery, chemotherapy, X-ray and ultrasound services, laboratory services and 24-hour emergency services. On average, 5-7 new patients with cancer present to the Cancer Center every week, and receive care at the hospital. Operating rooms and Intensive Care Unit have been established. Conclusion: Nepal's cancer incidence rate for males was 304 and for females was 333 per 10,000 in 2013, a significant increase from 128 and 151 per 10,000, respectively, in 2003 when the country's first hospital-based cancer registry was started. The incidence rate is predicted to increase to 38.5 and 41.4 per 100,000, respectively, by 2020 . It is critical to establish new cancer centers to deal with the surging burden of cancer in Nepal.

\section{8}

\section{AFRICAN-AMERICANS WITH MULTIPLE MYELOMA RECEIVE SHORTER DURATIONS OF FRONT-LINE THERAPY}

Mark A. Fiala, Tanya M. Wildes and Ravi Vij

Washington University School of Medicine, St Louis, MO, U.S.A.

Background: African-Americans with multiple myeloma (MM) have a favorable genetic profile but have inferior outcomes at the population level. This has largely been attributed to inferior access to newer therapies and stem cell transplantation. In this study, we also found that AfricanAmericans who receive these therapies receive shorter durations than their White peers do. Materials and Methods: All MM cases from 2007-2013 in the Surveillance, Epidemiology, and End Results-Medicare were reviewed along with their corresponding claims data through 2014. Cases not enrolled in Medicare Part A, B, and D; health maintenance organization enrollees; those diagnosed prior to age 65 years; and those surviving less than 6 months were excluded. The study population was limited to patients who received bortezomib/lenalidomide for first-line therapy as 
these drugs largely represented the standard of care for treatment-eligible patients during the study period. The number of doses provided within the first 6 months of treatment were compared. Results: A total of 2,313 patients were included in the analysis. The median age at MM diagnosis was 74 (range $=65-96$ ) years and $51 \%$ were male; $80 \%$ were White, $14 \%$ African-American, and $6 \%$ another race; $38 \%$ received bortezomib, $32 \%$ lenalidomide, and $30 \%$ both agents. African-Americans treated with bortezomib received a mean of 14.6 doses $(\mathrm{SD}=11.7)$ compared to 17.3 doses $(\mathrm{SD}=11.0)$ for the rest of the population $(p=0.0091)$. Those treated with lenalidomide received a mean of 104.4 doses ( $\mathrm{SD}=43.9)$ compared to $116.5(\mathrm{SD}=52.6 ; p=0.0208)$. Similar trends were observed among patients receiving combination therapy but were not statistically significant. Conclusion: African-Americans were found to receive shorter courses of front-line therapy; this likely contributes to the poorer outcomes observed at the population level. It is currently unclear if the shorter durations are associated with patient-related factors such as disease severity or comorbidities, or due to access issues. Additional analyses are planned to help determine this.

\section{9}

\section{PATIENT ADVOCACY APPROACHES TO ADVANCING ACCESS TO CARE FOR WOMEN'S CANCER IN EAST AFRICA}

Darya A. Kizub ${ }^{1}$, Julie R. Gralow ${ }^{2,3}$, JoAnne Zujewski ${ }^{4}$ and Allison L. Dvaladze ${ }^{2,3}$

${ }^{1}$ The Everett Clinic, Everett, WA, U.S.A.;

${ }^{2}$ University of Washington, Seattle, WA, U.S.A.;

${ }^{3}$ Seattle Cancer Care Alliance, Seattle,

Washington, U.S.A.;

${ }^{4}$ Leidos Biomedical Research, Inc., Frederick, MD, U.S.A.

Background: Breast and cervical cancer are the most common cancer types among women in East Africa, where mortality remains high due to late presentation and limited access to treatment. Advocacy has played a key role in advancing cancer care in high-income settings. We examined local approaches to advocacy for women's cancer in East Africa to identify existing strengths and opportunities for improvement. Materials and Methods: Focus group discussions and in-depth interviews were conducted with a purposeful sample of participants who attended the $2016 \mathrm{WE}$ CAN summit in Nairobi, Kenya. Discussions were audiorecorded, transcribed, coded, and analyzed for prevailing themes. Results: Four focus groups and one in-depth interview were conducted with 51 participants. Thirty-six (70\%) participants identified as advocates, $30(59 \%)$ as cancer survivors, $14(27 \%)$ as non-governmental organization representatives, $13(25 \%)$ as researchers, four
$(8 \%)$ as clinicians, and six (8\%) as government/ policymakers. Most organizations focused on raising awareness, screening/early detection, and fundraising for treatment. Some participants raised concerns about the ethics of screening when treatment was out of reach and advocated for a broader strategy to improve access to treatment. Others described successful multi-sectoral collaborations between advocates, ministries of health, and international cancer organizations that catalyzed the adoption of and increased funding for national cancer control plans in Ethiopia and a country-wide screen and treat program for cervical cancer in Zambia. Still, coalitions remained the exception. Barriers to advocacy included limited funding, difficulty approaching policymakers, limited collaboration with medical personnel, and difficulty establishing a coalition. Conclusion: Advocacy for women's cancer in East Africa is still in its early stages, yet there is a demand from advocates for tools, training, and evidence-based solutions. Multi-sectoral collaboration and coalitions, both in-country and acrossborders, are crucial to sharing experiences and developing local solutions to common barriers.

\section{0}

\section{THE BLUE IN THE PINK: MALE BREAST CANCER SURVIVOR STORIES}

Marya Shegog ${ }^{1}$, Axenya Kachen ${ }^{1}$, Alexandria Evans ${ }^{1}$ and Raheem Paxton ${ }^{2}$

${ }^{1}$ University of Nevada, Las Vegas, School of

Public Health, Las Vegas, NV, U.S.A.;

${ }^{2}$ The University of Alabama, College of Community

Health Sciences, Institute for Rural Health

Research, Tuscaloosa, AL, U.S.A.

Background: Men account for fewer than $1 \%$ of all breast cancer diagnoses in the United States, and the survivors belong to a small and underserved population $(1,2)$. Due to the significant increase of incidence among women, as well as an emphasis on research, treatment, and cures for women, breast cancer has a 'feminized' pathology $(1,3)$. The diagnosis, treatment, and survival among men who experience breast cancer has not been fully explored, and therefore awareness, diagnosis and treatment for targeting men is absent from the Public Health discourse. Materials and Methods: This study utilized mixed-methods analysis using MAXQDAv12 software to analyze stories $(n=134)$ told by male breast cancer survivors and their care givers. The stories were voluntarily submitted through a web portal to a nonprofit organization dedicated to male breast cancer awareness that is in an ongoing partnership with the University of Alabama. Institutional Review Board Exempt Status was provided for secondary analysis, through the University of Nevada, Las Vegas and the University of Alabama. Results: 
Table I. Temporal trends in time from abnormality to biopsy and treatment in 2015 to 2018 .

\begin{tabular}{lccccc}
\hline & 2015 & 2016 & 2017 & 2018 & $2015-2017$ \\
\hline $\begin{array}{l}\text { Total } \\
\text { Screening }\end{array}$ & 8 & 26 & 42 & 28 & 76 \\
$\quad$ Detected, n & $4(0.5)$ & $5(0.19)$ & $18(0.43)$ & $9(0.32)$ & $27(0.36)$ \\
Mean time, days & & & & 22 & 28 \\
$\quad$ Screening to complete Dx imaging & 32 & 32 & 23 & 38 & 24 \\
$\quad$ Dx imaging to biopsy & 23 & 30 & 33 & 33 \\
$\quad$ Diagnosis to therapy initiation & 40 & 30 & & 25 \\
\end{tabular}

The stories revealed an alarming rate of missed or delayed diagnoses $(25 \%)$ resulting in deferral of treatment and possible further health complications. How men navigated the femalecentered breast cancer system was dependent on several factors including: Diagnosing clinician, access to care, support networks and individual's knowledge and perceptions of the disease. Conclusion: Men who survived breast cancer had unique stories of diagnosis, treatment, and ultimately survival; however, the commonalities across their stories revealed the need for male breast cancer awareness, education, and improved patient-centered care that is gender specific.

1 Giordano SH, Buzdar, AU and Hortobagyi GN: Breast cancer in men. Ann Intern Med 137: 678-687, 2002. PMID: 12379069. DOI: 10.7326/0003-4819-137-8200210150-00013

2 Gómez-Raposo C, Tévar FZ, Moyano MS, Gómez ML and Casado E: Male breast cancer. Cancer Treat Rev 36: 451457, 2010. PMID: 20193984. DOI: 10.1016/j.ctrv.2010. 02.002

3 Ly D, Forman D, Ferlay J, Brinton LA and Cook MB: An international comparison of male and female breast cancer incidence rates. Int J Cancer 132: 1918-1926, 2013. PMID: 22987302. DOI: $10.1002 /$ ijc. 27841

\section{1 \\ MAMMOGRAPHIC SCREENING OF AN UNDERSERVED MEDICAL POPULATION}

Annie Tang, Shannon Ugarte, Zhonnet Harper, Kevin B. Knopf and Amal Khoury

Highland Hospital, Oakland, CA, U.S.A

Background: Early detection and treatment of breast cancer is the cornerstone of current practice. Despite improvement in screening over the last several decades, women of lower socioeconomic status have not seen the same benefit as women of high socioeconomic status. Materials and Methods: In order to understand and remedy barriers to timely screening, diagnosis and initiation of therapy, we created a prospective database of patients with breast cancer at our urban medical center. Satellite community offices staffed with primary care providers and OBGYN physicians obtained mammography per screening guidelines. Patients were followed-up longitudinally in order to track time to completion of diagnostic work-up and time to initiation of therapy. We also retrospectively evaluated patients who were diagnosed with breast cancer from 2015-2017 to understand trends over time. Results: We identified 28 patients who were diagnosed/treated in 2018 (Table I). Of those 28, only 11 (32\%) were diagnosed based on a screening mammogram, the remaining $68 \%$ presented with a mass, pain, or nipple discharge. Of the 76 patients diagnosed between 2015-2017, only 27 cancer cases $(36 \%)$ were screen-detected. The average time between initial screening and completion of diagnostic imaging was 21.6 vs. 28 days in 2018 vs. 20152017 , respectively. The average time between diagnostic imaging and biopsy was equal (24 days). The average time between diagnosis and the initiation of any treatment (local or systemic) was 36 vs. 33 days, respectively. Conclusion: Efficiency of care is achievable in a safety- net hospital.

\section{2}

\section{IMPLEMENTATION OF IMPROVED BREAST CANCER RECEPTOR SUBTYPING IN NIGERIAN CLINICAL PRACTICE}

Ibraheem F. Abiola, Polite Blasé and Olopade Olufunmilayo

Section of Hematology Oncology, University of Chicago, IL, U.S.A.

Background: Breast cancer (BC) is a common cancer in Nigeria with a high mortality rate. In Nigeria, receptor status assessment for patients with BC is not standard of care; patients who are surgical candidates undergo total mastectomy with or without sentinel node biopsy, followed by chemotherapy with or without tamoxifen. The importance 
of $\mathrm{BC}$ receptor subtyping for prognosticating disease outcome and predicting therapy options is not emphasized. We therefore assessed the use of BC subtyping in clinical practice. Materials and Methods: To explore these barriers, particularly in Lagos with a higher socioeconomic status, a pilot study was conducted to assess the rate of BC subtyping in newly diagnosed patients. Data collected from a public laboratory at Lagos State Teaching Hospital (LASUTH) and three major private laboratories over a 3- to 6-month period). Results: At LASUTH, over a 3-month period (March-May 2018) only $27 \%$ of 74 new BC cases underwent BC subtyping prior to treatment. At the private laboratory, over a 6-month period (November 2017-May 2018), only $18 \%$ of 289 new BC cases underwent subtyping prior to treatment. Perceived barriers postulated by pathologists and laboratory technicians/scientists were: (i) The direct cost of IHC to patients (N35,000 at LASUTH and N45,000-90,000 at a private laboratory, that is $\times 97$ to $\$ 125$ ); (ii) patients were given a choice by their physicians without knowing the implication of their choices; (iii) not all physicians ordered $\mathrm{BC}$ subtyping (especially less in the private sector); (iv) the time for reporting the results was too long i.e. ranged from 2-4 weeks. Conclusion: Implementation strategies in ensuring evidenced-based practices in low- and middle-income countries is of urgent concern. It is not enough to supply infrastructure or educate the workers in these environs, the use of implementation science in global oncology must also be expanded in order to maximize resources.

\section{3 \\ PATTERNS OF LAXATIVE PROPHYLAXIS FOR OPIOD-INDUCED CONSTIPATION IN VETERANS WITH LUNG CANCER ON FIRST OPIOID PRESCRIPTION}

Arjun Gupta, Hui Yang, Deepak Agrawal, David Gerber and Carlos Alvarez

Sidney Kimmel Comprehensive Cancer Center, Johns Hopkins University, Baltimore, MD, U.S.A.
Background: Opioid-induced constipation (OIC) is the most common side-effect in patients prescribed opioids for cancer pain treatment. Oncology and gastrointestinal guidelines recommend routine prescription of a laxative agent for preventing OIC in all patients prescribed an opioid unless a contraindication (such as pre-existing diarrhea) exists. One of the commonly prescribed laxatives is docusate, although studies have demonstrated its inefficacy. We explored patterns of prescription of laxative agents in patients with lung cancer for first opioid prescription. Materials and Methods: This was a retrospective, observational study evaluating the prescription of laxatives for OIC to adult patients with incident lung cancer (ICD-9 codes 162.0-162.9 and ICD-10 code C34.00-34.90) in the Veteran's Affairs (VA) Corporate Data Warehouse (CDW), with first opioid prescription between January 1, 2003, and December 31, 2016. The CDW includes inpatient and outpatient diagnosis codes and pharmacy data. Data were analyzed using the VA Informatics and Computing Infrastructure. Exposure to laxative agents was categorized as: None provided, docusate alone, docusate plus another laxative, and other laxatives only. Prescriptions for laxatives within 1 week of the opioid prescription were considered as prophylaxis. Results: Overall, 130,990 individuals were included in the analysis: 97.5\% men, $73.2 \%$ White and $14.5 \%$ Black. Of these, $98,206(75 \%)$ received no prophylaxis, 16,129 (12.3\%) received docusate alone, 6,650 $(5.1 \%)$ received docusate with another laxative, and 9,990 (7.6\%) received laxative(s) other than docusate. In total, $87.3 \%$ of patients received inadequate prophylaxis (none or only docusate) and $5.1 \%$ received unnecessary addition of docusate to another laxative. Conclusion: In this large retrospective study of veterans with lung cancer, $75 \%$ of the patients receiving opioids, contrary to guidelines, were not prescribed any laxative. Even when laxative prophylaxis was prescribed, it was inappropriate (only docusate, or docusate with another laxative) in two-thirds of patients. Further investigation into reasons for inappropriate OIC prophylaxis practice patterns is warranted. 